\title{
Non-odontogenic hard palate cysts with special reference to globulomaxillary cyst
}

\author{
${ }^{1}$ Department of Radio-diagnosis, SGT Medical College, Gurgaon 122505, India. \\ ${ }^{2}$ Department of ENT \& Head \& Neck Surgery, PGIMER \& RML Hospital, New Delhi 110001, India. \\ ${ }^{3}$ Department of ENT \& Head \& Neck Surgery, SGT Medical College, Gurgaon 122505, India. \\ ${ }^{4}$ Department of Orthodontics, Sri Guru Ramdas Dental College, Amritsar 143006, India. \\ ${ }^{5}$ Department of Orthodontics, Himalyan Institute of Dental Sciences, Paonta Sahib 173025, India. \\ ${ }^{6}$ Department of Oral \& Maxillofacial Surgery, Deepak Dental Centre, Pathankot 145001, India.
}

Bharat Bhushan Sharma ${ }^{1}$, Shweta Sharma ${ }^{2}$, Arvind $\mathrm{Jha}^{3}$, Kamal Deep Sharma ${ }^{4}$, Jai Deep Sharma ${ }^{5}$, Chattur Bhuj Sharma ${ }^{6}$

Correspondence to: Dr. Bharat Bhushan Sharma, Department of Radio-diagnosis, SGT Medical College, Gurgaon 122505, India. E-mail: bbhushan986@gmail.com

How to cite this article: Sharma BB, Sharma S, Jha A, Sharma KD, Sharma JD, Sharma CB. Non-odontogenic hard palate cysts with special reference to globulomaxillary cyst. Plast Aesthet Res 2016;3:302-5.

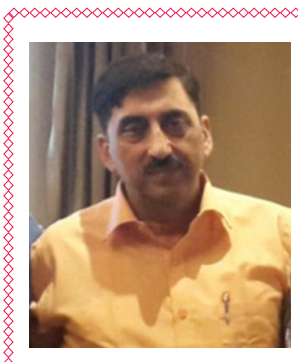

Dr. Bharat Bhushan Sharma is Professor \& Head of Department of Radio-diagnosis, SGT Medical College, Gurgaon, India. He did Post Graduation from International Reputed Institute of PGIMER, Chandigarh, India. He then served in Army Medical Corps and later served in HP State Health Services and Central Health Services as consultant radiologist. He started different radiological services modalities like CT and MRI first time in Himachal Pradesh Health Services in the state. He has been teaching post graduates for the last 25 years and conducted various radiological research projects of Post Graduates \& PhD students during 1995-2015. He has around 60 publications (national \& international) at his credit. He joined the present institute on 01.05.2015 and got installed the latest modern radiological modalities to start Post Graduation in radio-diagnosis. Beside medical courses, he is also engaged in teaching allied health science courses, like Diploma in Radio Imaging Technology (DRIT), Bachelor of Radio Imaging Technology (BRIT) and Master of Radio Imaging Technology (MRIT) in the department.

\section{Article history: \\ Received: 19-05-2016 \\ Accepted: 02-09-2016 \\ Published: 20-09-2016 \\ Key words: \\ Palatal cyst, \\ globulomaxillary cyst, \\ non-odontogenic, \\ magnetic resonance imaging, \\ computerized tomography}

\section{ABSTRACT}

Palatal cysts are always confusing by defining their exact nomenclature or conclusive diagnosis. One of these presentations is globulomaxillary cyst which requires to be categorized under appropriate head for the management point of view. Though this entity appears to be of odontogenic in origin but because of its anatomical relation and histo-pathological background this is placed in non odontogenic group. Though the mechanism of its formation remains the same but this cyst cannot be mixed up with nasopalatine cyst as per their location. Globulomaxillary cyst appears as inverted pear shaped radiolucency in all radiological procedures. This remains asymptomatic for a long time and rarely gets infected. We present a 29-year-old male who reported with one year history of asymptomatic right side hard palate swelling. He was subsequently diagnosed as globulomaxillary cyst with the help of radiological modalities like computerized tomography and magnetic resonance imaging. This article will highlight mainly the clinical and radiological features of these cysts with particular reference to globulomaxillary cyst which is our presenting case.

(i) (2) This is an open access article distributed under the terms of the Creative Commons Attribution-

BY NC SA NonCommercial-ShareAlike 3.0 License, which allows others to remix, tweak, and build upon the work non-commercially, as long as the author is credited and the new creations are licensed under the identical terms. 


\section{INTRODUCTION}

Cysts in the oral cavity can either be of soft tissue origin or from within the bone. Non-odontogenic hard palate cysts arise from the tissues which do not participate in tooth formation. There are many palatal cysts and their variants are encountered during the course of embryonic palate development. One of the cysts is globulomaxillary cyst and this terminology had a lot of dispute to be used. It was earlier thought to be of embryonic origin because of entrapment of the ectoderm but now this hypothesis is no longer considered. These have been considered as fissural entrapment of epithelium rather than embryonic ectoderm. ${ }^{[1]}$ There are many other cysts reported in the palate region and have been categorized as per the origin and anatomical location.

\section{CASE REPORT}

A 29-year-old male reported to otolaryngology outpatient department with the swelling in the hard palate of one year duration [Figure 1].

This was asymptomatic in the beginning but subsequently developed some roughness along with slight local tenderness. There was no history of trauma or fever. On examination there was slight protuberance over the right side of the hard palate. This was of slight dull pink in coloration. There was no ulceration seen locally over the swelling. There was no divergence of roots of central incisors. The adjoining teeth reacted normally to the electric vitalometer test and to temperature stimulation. All the biochemical parameters were within normal limits. The further detailed initial work up confirmed the swelling as that of the non odontogenic origin. The patient was referred for evaluation of the tumor. The oral occlusal film had confirmed the swelling of non odontogenic origin. The patient was subjected to plain as well as contrast enhanced computerized

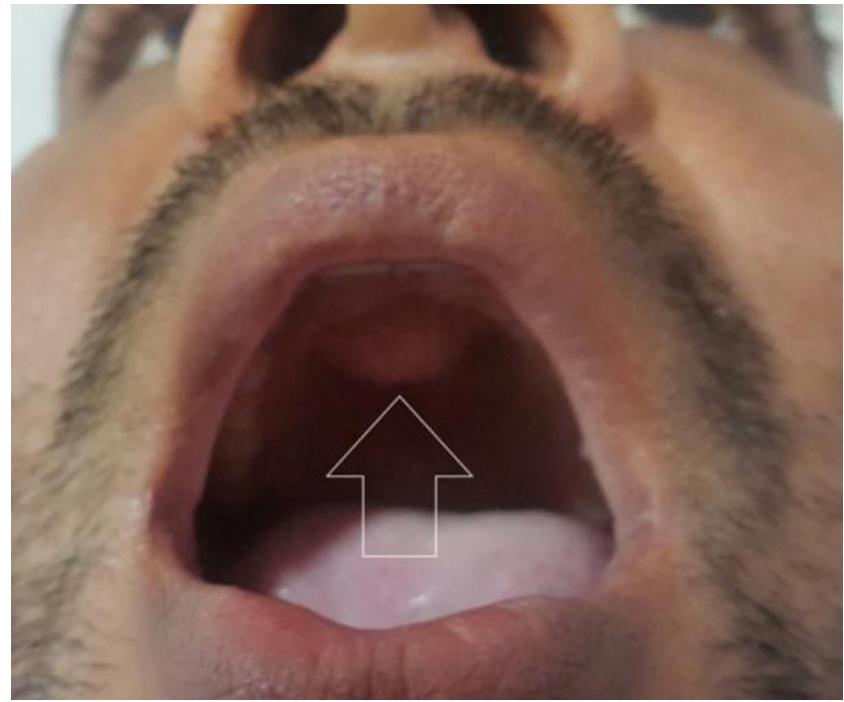

Figure 1: A 29-year-old male with hard palate swelling which is slightly pink in coloration (white vertical arrow)

tomography (CT) scanning of the face and neck region. The findings revealed a radiolucent pear shaped non enhancing unilocular, inverted pear shaped cystic lesion $2.5 \mathrm{~cm} \times 3.5 \mathrm{~cm}$ in size between the lateral incisor and canine on right side [Figure 2].

There was also thinning out of the bony outline [Figure 3].

Patient was also subjected to magnetic resonance imaging (MRI) scanning of face and neck region. The mass was of fluid density which was hypointense in T1W and hyperintense in T2W images. There were no associated findings [Figure 4].

Fine needle aspiration cytology has shown some seropurulent secretion and confirmed as non odontogenic cyst coming in the category of globulomaxillary cyst. The histo-pathological images were not available. The patient has been planned for
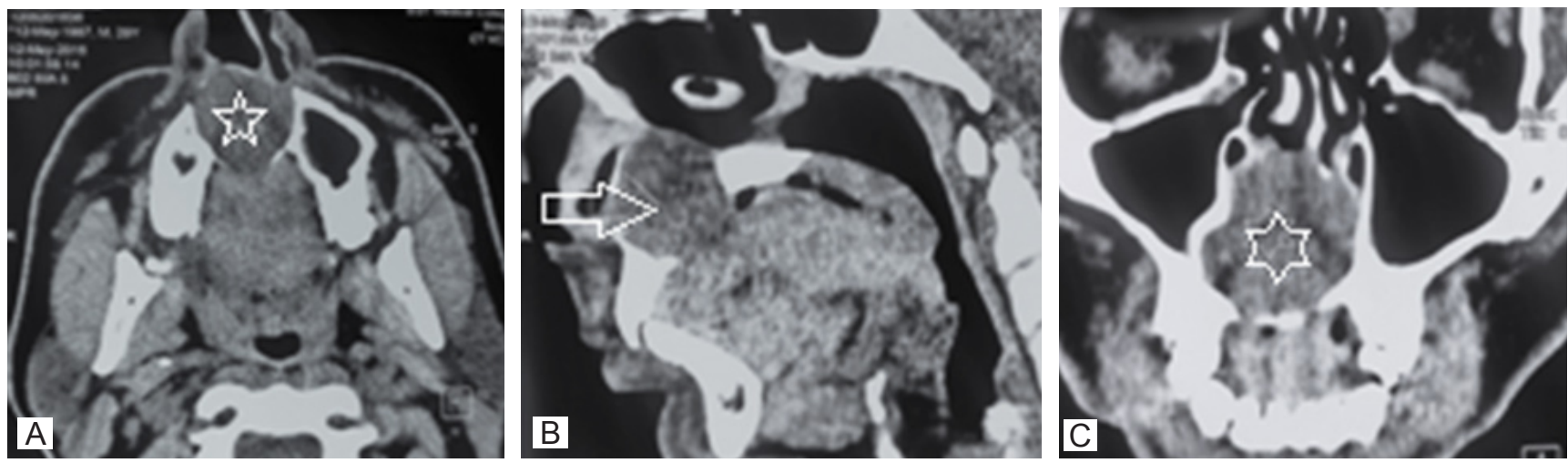

Figure 2: Contrast enhanced computerized tomography of face and neck region. (A) Axial section shows a radiolucent non enhancing lesion (white star) present on right side of the hard palate; (B) reformatted sagittal section shows the same as pear shaped lesion (horizontal white arrow) abutting the right nasal cavity; $(\mathrm{C})$ reformatted coronal section shows the lesion is encroaching upon the right maxillary sinus (white star) without invading the same 

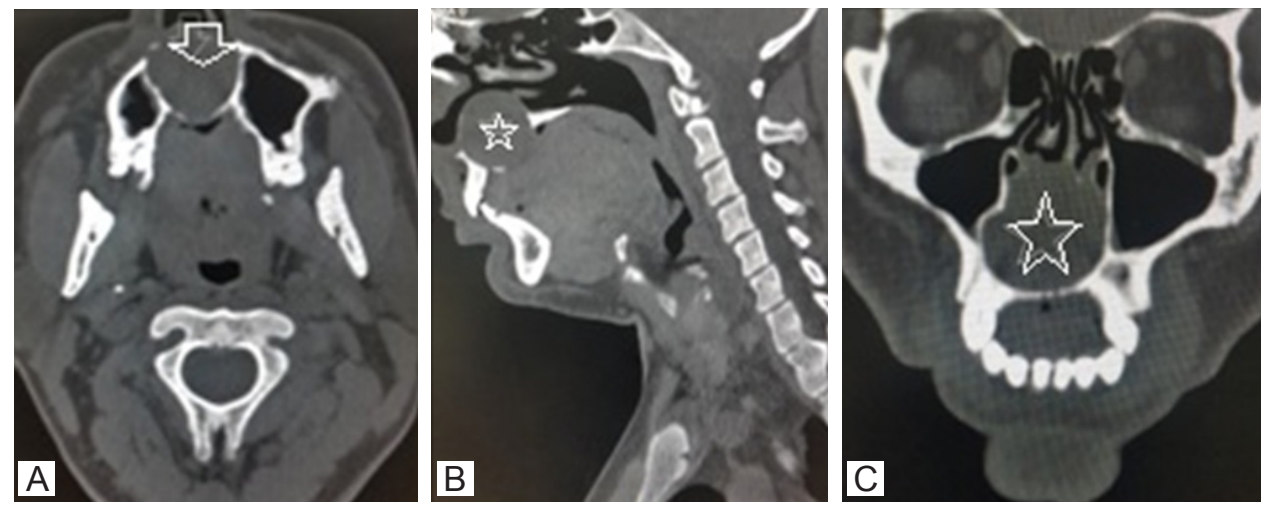

Figure 3: Non contrast computerized tomography of facial and neck region in 3D reformatted bone window. (A) Axial section shows expanded radiolucent lesion with "egg shell" appearance at the base of nasal region predominantly on right side (vertical white arrow); (B) sagittal section shows the superior extent of lesion from hard palate (white star); (C) coronal section delineate the lesion separate from the maxillary sinuses but obscuring the nasal passages predominantly on right side (white star)
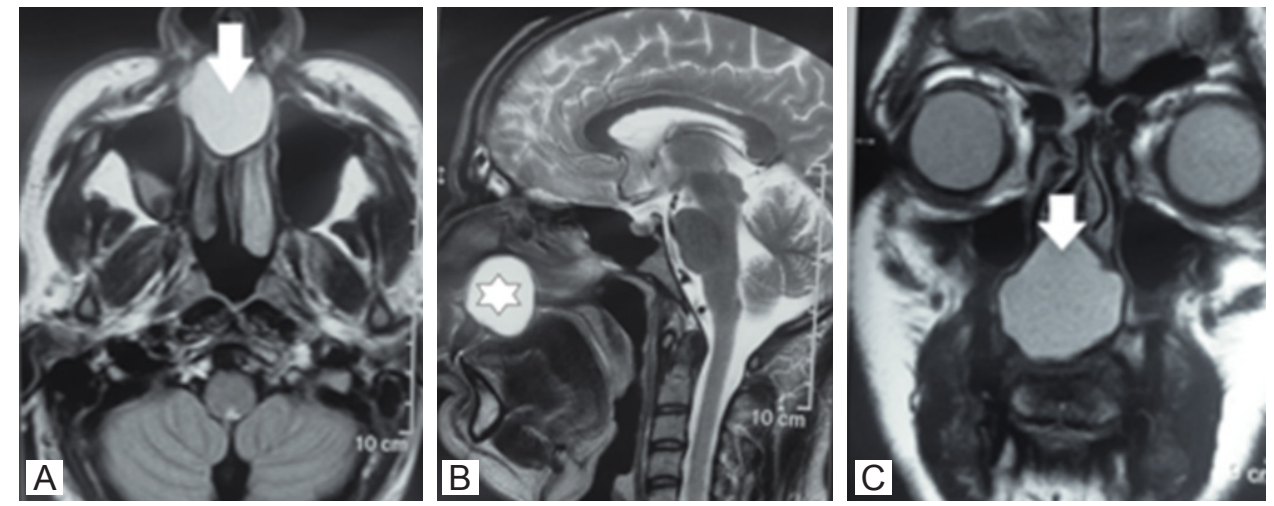

Figure 4: Plain magnetic resonance imaging face and neck region. (A) T2W image shows the well contained hyperintense cystic lesion on right side of the hard palate (vertical arrow); (B) T2W sagittal image shows hyperintense well demarcated lesion on the superior part of the hard palate (white star) on right side; (C) same lesion in T2WI coronal section with its superior extent (vertical arrow)

enucleation of the cyst as this being the most preferred method of treatment of these entities.

\section{DISCUSSION}

Hard palate forms the floor of the nostrils and roof of the oral cavity. This is thicker in front and thin in its posterior aspect. Globulomaxillary cysts are still disputable in their origin but majority of studies have shown these as not of embryonic origin. ${ }^{[2]}$

Oral origin cysts can be placed in to two categories as follow: (1) non-odontogenic (fissural) category includes globulomaxillary, nasopalatine, median palatal and nasolabial (nasoalveolar) cysts; and (2) odontogenic category includes dentigerous cysts, primordial cysts, odontogenic keratocysts and residual cysts.

Globulomaxillary cysts fall in non-odontogenic category. These can be distinguished based on the origin of the epithelial rests. Odontogenic cysts arise from tooth developing epithelium contrary to non odontogenic which arise from the trapped epithelium because of the fusion of upper jaw bones..$^{[3]}$ Non-odontogenic cysts can further be differentiated on the basis of their anatomical location. All the cysts around incisive canal fall in this group [Figure 5].

Globulomaxillary cysts arise at the junction between maxilla and premaxilla. There are three main subtypes of non-odontogenic fissural cysts described as nasoalveolar, nasopalatine and median palatal. ${ }^{[4]}$ These are usually discovered during routine clinical or radiographic examinations. The average duration of these cysts vary from one week to two years. The occlusal images are the first to lead in radiological investigations. In plain X-ray this may present as radiolucent region. These are usually asymptomatic and also found as incidental findings in CT examinations. Their pathogenesis though controversial but non disintegrated epithelium in the fissural sites remains the most accepted hypothesis. These are usually painless and rarely get infected and that is the reason for their delayed diagnosis. ${ }^{[5]}$ These are oval or round and hypodense on CT examination and do not enhance in post contrast studies. Bone resorption is often present 


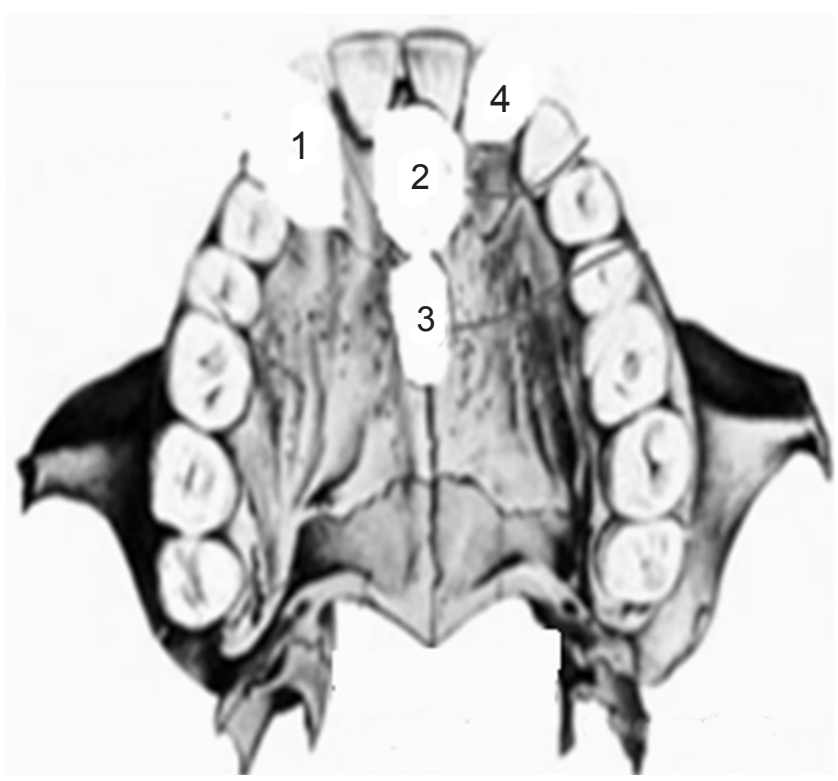

Figure 5: Diagrammatic representation of hard palatal cysts as per locations. Globulomaxillary cyst (1), nasopalatine cyst (2), median palatal cyst (3) and nasolabial cyst (4) are shown as white oval regions

because of their expansile nature but the cortical break is very rare. The classical egg shell type of margins is present as was also seen in our case. Hisatomi et al. ${ }^{[6]}$ has described the classical features of these cysts in MRI. MRI show intermediate signal in T1W and hyper intensity on $\mathrm{T} 2 \mathrm{~W}$ images. There is no post-gadolinium enhancement seen in T1W fat saturated sequences. Histologically these present as a cystic cavity covered by epithelium that contain stratified squamous cells with some respiratory epithelial components. The wall is composed of thick dense collagenous fibrous tissues. Median palatine cysts are easy to diagnose on CT and MRI modalities as their locations are self explanatory. ${ }^{[7,8]}$ There are cases where these entities have been managed with non-surgical endodontic treatment ${ }^{[9]}$ but the gold standard is complete surgical excision via intraoral approach. ${ }^{[10]}$ Sometimes the part of nasal mucosa has to be sacrificed as the lesion is close to the floor of the nose. ${ }^{[11]}$

In conclusion, globulomaxillary cysts draw attention only after these are diagnosed. These cysts remain undiagnosed for a long period because of their asymptomatic background. CT and MRI modalities have brought revolution in diagnosing these entities while performed for some other reasons. These should be classified in their proper category before treating them.

\section{Financial support and sponsorship None.}

\section{Conflicts of interest}

There are no conflicts of interest.

\section{Patient consent}

The consent of the patient was taken before subjecting the patient for investigation.

\section{Ethics approval}

The approval for publishing this case and paper had been obtained from the institute.

\section{REFERENCES}

1. Haring P, Filippi A, Bornstein MM, Altermatt HJ, Buser D, Lambrecht JT. The "globulomaxillary cyst" a specific entity or a myth? Schweiz Monatsschr Zahnmed 2006;116:380-97. (in French, German)

2. Dammer U, Driemel O, Mohren W, Giedl C, Reichert TE. Globulomaxillary cysts--do they really exist? Clin Oral Investig 2014; 18:239-46

3. Alimendinger A, Gabe M, Destian S. Median palatine cyst. Neuroradiology 2009;3:7-10.

4. De Cuyper K, Vanhoenacker FM, Hintjens J, Verstraete KL, Parizel PM. Nasopalatine duct cyst. JBR-BTR 2008;91:179.

5. Yerli H, Cabbarpur C, Aydin E. CT findings of a nasoalveolar cyst. $B r J$ Radiol 2009;82:e76-8.

6. Hisatomi M, Asaumi J, Konouchi H, Shigehara H, Yanagi Y, Kishi K. MR imaging of epithelial cysts of the oral and maxillofacial region. Eur J Radiol 2003;48:178-82.

7. Karacal N, Ambarcoglu O, Kutlu N. Median palatine cyst: report of an unusual entity. Plast Reconstr Surg 2005;115:1213-4.

8. Hadi U, Younes A, Ghosseini S, Tawil A. Median palatine cyst: an unusual presentation of a rare entity. Br J Oral Maxillfac Surg 2001;39:278-81

9. Abdel-Azim MM. Healing of globulomaxillary cyst after non-surgical endodontic treatment--“case report". Egypt Dent J 1995;41:1295-8.

10. Fonseca R, Marciani R, Turvey T, editors. Oral and Maxillofacial Surgery. 2nd ed. St. Loius: Elsevier; 2008. p. 418-65.

11. Hupp JR, Ellis E, Tucker MR. Contemporary oral and maxillofacial surgery. 5th ed. St. Louis: Mosby Elsevier; 2008. p. 450-6. 